\title{
A new strategy for total auricular reconstruction using prelamination of extended retroauricular flap with tissue \\ expansion
}

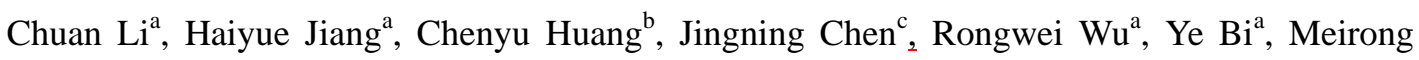
Yang $^{\mathrm{a}}$, Ye Zhang ${ }^{\mathrm{a}}$, Bo Pan ${ }^{\mathrm{a}}$

a, Center of Auricular Reconstruction, Plastic Surgery Hospital, Peking Union Medical College, Badachu Road No.33, Beijing, People's Republic of China;

b, Department of Plastic, Reconstructive and Aesthetic Surgery, Beijing Tsinghua Changgung Hospital; Medical Center, Tsinghua University, Beijing 102218, China.

c, Department of ENT, China-Japan Friendship Hospital, Cherry Park Street No. 2, Beijing, People's Republic of China.

Corresponding author: Bo Pan, Center of Auricular Reconstruction, Plastic Surgery Hospital, Peking Union Medical College, 33 Badachu Road, Beijing, People's Republic of China.

Telephone: +86-18813019675, E-mail: 15098733987@126.com

\section{Summary}

Background: To accomplish total ear reconstruction with aesthetic appearance is a great challenge for plastic surgeons worldwide due to insufficient skin coverage. A retroauricular fascia flap and skin graft technique are commonly used, but the results are unsatisfied due to color mismatch, severe edema, and donor site morbidity. Here, we will describe a novel strategy for total ear reconstruction, utilizing an extended retroauricular flap prelaminated with tissue expansion to obtain enough skin for ear reconstruction.

Methods: About two months before ear reconstruction, kidney shaped tissue expander was inserted at the mastoid region subcutaneously. The retroauricular skin became enlarged and thinner in 2 months after expansion. Next, the expander was removed and the retroauricular flap was extended by dissecting the surrounding scalp subcutaneously. By mobilizing the scalp-extended retroauricular flap, we could encapsulate the entire framework with thin and non-hair bearing skin.

Results: From August 2014 to September 2015, 36 microtia patients had undergone ear reconstruction using the novel strategy. All cases have resulted in satisfactory aesthetics with fine structure, symmetry, maintenance of the auriculocephalic angle.

Conclusions: Using our novel strategy, we can obtain satisfactory aesthetic outcome by fully mobilizing the expanded skin without additional morbidities of donor sites. Patients are satisfied with the vivid ear contour and lack of groin scar.

Keywords: microtia; ear reconstruction; flap prelamination; tissue expansion; skin grafts

\section{Introduction}

Total auricular reconstruction is one of the greatest challenges for plastic surgeons worldwide. A sufficient skin envelope and sophisticated auricular framework are two important factors to a successful auricular reconstruction ${ }^{[1-3]}$. Tissue expansion will theoretically provide additional soft tissue to cover the cartilage framework in creating a three-dimensional and contour-attenuated ear $^{[4-6]}$. During tissue expansion procedure, the superior match of color and texture along with 
recovery and maintenance of sensations need to be well considered for plastic surgeries including auricular reconstruction. However, the expanded skin with such merits is not enough for the coverage of the posterior surface of the framework for total auricular reconstruction, so retroauricular fascia flap and skin grafts are usually adopted. In addition, patients are not satisfied with the donor site mobility and color mismatch ${ }^{[7]}$.

Flap prelamination is a process of bonding of distinctive layers to create a novel anatomic three-dimensional laminated structure without interfering with the native axial blood supply ${ }^{[8,9]}$. This process is widely used in complicated reconstructions where conventional methods are not applicable ${ }^{[10-12]}$. In order to envelop the entire framework with enough thin skin, we invented an extended retroauricular flap, which is consisted of central mastoid skin and surrounding scalp. In addition, we prelaminated the flap through tissue expansion to make the central non-hair bearing mastoid skin thinner and enlarged. In this paper, we will present the process and performance of our novel strategy on total ear reconstruction using the extended and prelaminated retroauricular flap.

\section{Patients and methods}

From August 2014 to September 2015, 36 congenital microtia patients had enrolled to undergo ear reconstruction using this new strategy in our center (Table1). In the patients, 23 were male and 13 were female, with ages of 5-30 years old (average 11.3 years old). Fourteen cases (33.3\%) were left-side microtia, 18 cases (46.6\%) were right-side microtia, and 4 cases (20\%) were bilateral microtia. According to Nagata's definition, 30 cases (80\%) were typical lobule-type microtia and 6 cases (20\%) were concha-type microtias.

\section{Surgical procedures}

Currently, we persist in using autogenous costal cartilage to make the framework for ear reconstruction. To achieve plenty skin to encapsulate the entire framework, the extended and prelaminated retroauricular flap has been used.

\section{Prelamination of extended retroauricular flap using tissue expansion}

To get enough skin to cover the entire framework in ear reconstruction, mastoid skin was prelaminated using soft tissue expansion. Briefly, a $3 \mathrm{~cm}$ incision line along the hairline was made on the scalp. A subcutaneous pocket was then dissected above the retroauricular fascia. Once strict hemeostasis was obtained, a 50-ml kidney-shaped expander was inserted to the subcutaneous pocket. The inflation valve was placed beneath the skin of the neck, and a negative pressure drainage device was inserted under the expander to prevent postoperative hematoma. The inflation began 7 days after operation. 4-6 $\mathrm{ml}$ saline was injected each time for 3 times per week until the expansion volume reached to $60-70 \mathrm{ml}$. The whole procedure took about 2 months.

After the expansion procedure was completed, we performed ear reconstruction. The first incision was about $3 \mathrm{~cm}$ long, along the posterior edge of the expanded skin. Through this incision, we got into the capsule and removed the expander. Then we used scalpel to dissect the surrounding scalp to $3 \mathrm{~cm}$ behind the hairline at subcutaneous level (Fig 1). The thickened capsule besides the edge of the expander was removed using ophthalmic scissors. Thus, the extended retroauricular flap was constructed: the central mastoid area was enlarged and thinner through tissue expansion; while the surrounding scalp made the central expanded skin much looser and 
transferable. The extended retroauricular flap obtained equal skin area as sub facial expansion with $80 \mathrm{ml}$ expander, however, the central skin are much thinner, which is much similar to normal ear skin (Fig 2-2).

\section{Ear reconstruction using prelaminated retroauricular flap and autogenous costal cartilage}

The ear framework fabricating method was performed as reported in our previous procedure ${ }^{[13]}$. Usually we harvested the sixth, seventh and eighth cartilage individually in children. The costal cartilages were carved and assembled with titanium wires to form 3-dimensional framework. The 3-dimensional framework is composed of three levels, including helix and crus of helix, the main body of the framework, and the base of the framework (Fig 3).

Next, the cartilage framework was inserted into the envelope. A negative pressure drainage device was placed beneath the flap. Because the surrounding scalp had been dissected and the restrictive capsule had been removed, the mobility of the expanded skin became much better. Thus, we could use the expanded non-hair bearing skin to encapsulate the entire framework, form cranio-auricular sulcus and cover partial mastoid area. We sutured the flap to temporal periosteum to form cranio-auricular sulcus. The surrounding scalp was pushed forward to cover the mastoid area. Transfixing sutures were used to prevent rebounding of the scalp. Finally, we made another incision on the remnant ear and adjacent expanded flap to accomplish ear lobe translocation. In this way, a vivid and protruding ear was reconstructed without retroauricular fascia flap elevation and skin grafts(Fig 2-3,4,5).

In 6-12 months after the reconstruction operation, we utilized the remnant ear to form tragus, and excised extraneous soft tissue to deepen conchal floor. The superior cranio-auricular sulcus was deepened with a small skin graft (about $3 \times 2 \mathrm{~cm}^{2}$ ) from the inguina.

\section{Results}

We carried out ear reconstruction with our novel strategy on 36 microtia patients. A follow up study has been done for 3-12 months. The reconstructed ears showed good three-dimensional configuration as well as good color match and texture. The entire surface of the reconstructed ear and most mastoid area has been resurfaced with the non-hair bearing skin flap. The cranioauricular angles of the reconstructed ears were similar to the opposite ears. Representative cases were presented in Figures 4, 5, 6.

Few complications occurred in our procedure, including exposure of tissue expander and skin loss (due to skin necrosis) over the framework (Table 2). Other complications, such as hematoma, dehiscence of incision, and infection, did not occur in our cases.

\section{Necrosis of coverage skin of the framework}

Because the expanded skin over the helix was apt to be oppressed after dressing, there were 2 cases of skin necrosis over the helix. The necrotic skin formed a thick layer of crust, so the vitality of the cartilage below was generally remained. The repairing operation was carried out 1 month after ear reconstruction when the grafting cartilage survived and swelling subsided.

First, we tried to apply thorough debridement to remove all the necrotic tissue. However, it was not easy to repair the wound due to the consideration of the exposure of the cartilage and the reoccurrence of the complications. Thus, we utilized retroauricular fascia and a double pedicle flap to cover the cartilage. The previous incision along the hairline was reused. Subcutaneous 
dissection was made from the incision to the edge of the wound to form a double pedicle flap which was consisted of ear dorsal skin and mastoid skin. Then, we elevated retroauricualr fascia which was $2 \mathrm{~mm}$ broader than the wound. The helix wound was first covered by the retroauricualr fascia, then the double pedicle flap was pushed to cover the fascia. This double-layer repairing guaranteed the safety, avoiding repeated repairing. The second wound caused by flap transferring was repaired with full thickness skin graft(Fig 7).

In addition, we made a $\mathrm{C}$ shaped thick dressing to protect the helix from oppression. Skin necrosis over the helix did not happen then (Fig 8).

\section{Exposure of tissue expander}

Exposure of tissue expander occurred in 3 cases. It was usually caused by the fold of tissue expander or vigorous inflation. The exposure area of the expander was about $1 \mathrm{~cm}^{2}$, and there was no purulent secretion beneath the expanded skin, so we performed ear reconstruction as usual. During the operation, the necrotic edge of skin defect was resected and the wound was sutured with nylon line and healed naturally (Fig 9).

\section{Discussion}

The auricle is one of the most sophisticated 3-dimensional structures of the external body. It contains more than 10 anatomical subunits, and its anterior skin is very thin and closely binds to perichondrium. Due to its complicated shape, ear reconstruction remains one of the greatest challenges for plastic and reconstructive surgeons worldwide. Since Gillies introduced total auricular reconstruction using autologous costal cartilage graft in 1920, many talented surgeons, such as Tanzer, Brent, Nagata, Firmin and Zhuang, made great advancement in the field of ear reconstruction ${ }^{[1,14]}$. The biggest progress has been relied on the improvement of framework fabrication. Though most reported fabrication methods can produce a vivid ear contour with multiple dimensions ${ }^{[15,16]}$, how to obtain enough thin skin to encapsulate the framework is still a great puzzle.

Due to its simplicity and convenience, tissue expansion has been widely used to produce soft tissue for the reconstruction of various defects. Since Newmann ${ }^{[17]}$ introduced tissue expansion in auricular reconstruction in 1957, more and more surgeons have applied tissue expansion to provide additional soft tissue for auricular reconstruction ${ }^{[18]}$. In our center, Jiang et a ${ }^{[13,19-21]}$ have reported successful microtia reconstruction using tissue expansion through a three-stage procedure. In their method, a $50 \mathrm{ml}$ kidney shape tissue expander is implanted subcutaneously. Through tissue expansion, non-hair bearing, thin and well-vascularized skin can cover the frontal side of an erect framework. Retroauricular fascia flap and skin grafts is used to cover the posterior surface of the framework. Residual ear is utilized to form tragus and elevate the framework in the third stage. In most expansion cases, additional harvest of skin graft is often required, which inevitably causes a donor-site scar and ear colour aberration. In addition, though enough tissue could be harvested through subfascial expansion, the shape of reconstructed ear is usually thick and clumsy due to the thickness of the flap ${ }^{[22-24]}$.

The term flap prelamination refers to the process of bonding of distinctive layers to create a novel anatomic three-dimensional laminated structure without interfering with the native axial blood supply ${ }^{[8,9]}$. It has been widely used in multiple complex conditions. Here, we have introduced prelamination of the extended retroauricular flap with tissue expansion to produce thin 
and soft tissue coverage for total ear reconstruction. The core concept of our strategy is prelaminating thin skin similar to normal ear skin and transferring the prelaminated skin to a proper position. The superior skin of ear is very thin and closely binds to the perichondrium, while the posterior skin is thicker and looser relatively. So we have prelaminated the mastoid skin with tissue expansion to make the skin thinner and enlarged, which strikingly helps exhibiting the details of ear structures. However, because the expanded flap is restricted by the thick fibrous capsule edge and surrounding scalp, it cannot be fully utilized to cover the entire framework. Then, we have dissected the surrounding scalp and removed the thick fibrous capsule edge to release the expanded skin. The area of dissection depends on the relaxation of the scalp, which is usually 3-4 $\mathrm{cm}$ wide. We have further advanced the surrounding scalp to the mastoid where transfixing sutures are used to prevent rebounding. In this way, the expanded skin become much looser, and it can be fully utilized to envelope the entire framework, forming cranio-auricular sulcus and covering partial mastoid region. The expanded non-hair bearing skin and surrounding scalp join together to constitute an intact flap, but with distinct function. Using this new strategy described here, we have obtained equal skin area as subfascial expansion with $80 \mathrm{ml}$ expander. More importantly, the skin enveloping the framework using the new strategy is much thinner, and the expansion period is shorter ( 2 months vs 4 months).

There are three key factors in our new technique:

1. The non-hair bearing skin of the mastoid region is limited. If the expander inserted subfascially is too large, the surrounding scalp would be expanded together and the expanded skin might be too thick to show the details of the framework. So we persist in inserting a $50 \mathrm{ml}$ kidney-shaped expander subcutaneously to produce enough thin skin.

2. In order to release the mobility of expanded skin, the surrounding scalp must be dissected and the thickfibrous capsule edge must be removed .

3. The $\mathrm{C}$ shaped dressing has to be used to protect the helix from oppression

In comparison to traditional $\mathrm{C}$ shaped retroauricular flap, the extended and prelaminated retroauricular flap get much better blood supply because most surrounded skin is not incised. So, the risk of flap necrosis due to blood supply shortage is lowed. In addition, the retroauricular fascia is remained intact. If skin necrosis over the framework happens, we could alternatively repair the wound with retroauricular fascia and free skin grafts.

\section{Conclusions}

Ear reconstruction using the extended and prelaminated retroauricular flap is easily manipulated. This new ear reconstruction strategy turns in better aesthetic outcome with rarer complications. In addition, skin graft is not required, which often leads to poorly matched skin color and creates additional groin scarring. Neither the addition of an operation procedure nor prolonged expansion is needed, so the patients are further satisfied with the shorter hospitalization.

\section{Conflicts of interest}

None declared.

\section{Ethical approval}

The study was approved by the Ethics Committee of the Plastic Surgery Hospital, Peking Union Medical College, and Chinese Academy of Medical Sciences. 


\section{Acknowledgements}

We thank all the patients for their participation and permission in this study. This work was supported by the Chinese National Science Foundation (No.81272724) and the Beijing Union Medical College Graduate Student Innovation Fund (No.10023-1002-2001).

\section{References}

1. Tanzer RC. Total reconstruction of the external ear. Plast Reconstr Surg Transplant Bull.1959;23:1-15.

2. Brent B. Auricular repair with autogenous rib cartilage grafts: two decades of experience with 600 cases. Plast Reconstr Surg.1992;90:355-374.

3. Kawanabe Y, Nagata S. A new method of costal cartilage harvest for total auricular reconstruction: part I. Avoidance and prevention of intraoperative and postoperative complications and problems. Plast Reconstr Surg.2006;117:2011-2018

4. Hata Y. Do not forget the fundamental merits of microtia repair using a tissue expander. Plast Reconstr Surg.2002;109:819-822.

5. Oneal RM, Rohrich RJ, Izenberg PH. Skin expansion as an adjunct to reconstruction of the external ear. Br J Plast Surg.1984;37:517-519.

6. Hata Y, Vmeda T. Reconstruction of congenital microtia by using a tissue expander. J Med Dent Sci. 2000; 47:105-116.

7. Nagata S. Secondary reconstruction for unfavorable microtia results utilizing temporoparietal and innominate fascia flaps. Plast Reconstr Surg. 1994;94:254-65.

8. Pribaz JJ, Fine N, Origill DP. Flap prefabrication in the head and neck: a 10-year experience. Plast Reconstr Surg. 1999;103:808-820.

9. Pribaz JJ, Guo L. Flap prefabrication and prelamination in head and neck reconstruction. Semin Plast Surg.2003;17:351

10. Pribaz JJ, Guo L. Clinical flap prefabrication. Plast Reconstr Surg.2009;124:340-350

11. Lazzeri D, SuW, Qian Y. Prefabricated neck expanded skin flap with the superficial temporal vessels for facial resurfacing. J Reconstr Microsurg. 2013;29:255-261

12. Topalan M, Guven E, Demirtas Y. Hemifacial resurfacing with prefabricated induced expanded supraclavicular skin flap. Plast Reconstr Surg. 2010;125:1429-1438

13. Jiang H, Pan B, Lin L, Cai Z, et al. Ten-year experience in microtia reconstruction using tissue expander and autogenous cartilage. Int J Pediatr Otorhinolaryngol. 2008;72:1251-9.

14.Tanzer, RC. Total reconstruction of the auricle: The evolution of a plan of treatment. Plast Reconstr Surg.1971;47:523.

15. Kim YS. The importance of a conchal bowl element in the fabrication of a three-dimensional framework in total auricular reconstruction. Arch Plast Surg. 2013;40:192-7.

16. Firmin F. State-of-the-art autogenous ear reconstruction in cases of microtia. Adv Otorhinolaryngol.2010;68:25-52.

17. Newmann CG.The expansion of an area of skin by progressive distention of a subcutaneous balloon; use of the method for securing skin for subtotal reconstruction of the ear. Plast Reconstr Surg (1946). 1957;19(2):124-30.

18. Hata Y, Hosokawa K, Yano K, et al. Correction of congenital microtia using the tissue expander. Plast Reconstr Surg. 1989;84:741-751 [discussion 52-53]. 
19. Zhuang HX, Jiang HY, Pan B, et al. Ear reconstruction using soft tissue expander in the treatment of congenital microtia. Zhonghua Zheng Xing Wai Ke Za Zhi.2006;22:286-289.

20. Dashan Y, Haiyue J, Qinghua Y, et al. Technical innovations in ear reconstruction using a skin expander with autogenous cartilage grafts. J Plast Reconstr Aesthet Surg.2008;61 Suppl 1:S59-69.

21. Pan B, Jiang H, Guo D, et al. Microtia: ear reconstruction using tissue expander and autogenous costal cartilage. J Plast Reconstr Aesthet Surg. 2008;61 Suppl 1:S98-103

22. Liu J, Sun J, Li X. Total auricular reconstruction without skin grafting. J Plast Reconstr Aesthet Surg.2011;64:1312-1317.

23. Park C. Subfascial expansion and expanded two-flap method for microtia reconstruction. Plast Reconstr Surg.2000;106: 1473-1487.

24. Park BY, Im JT, Lim SY. Microtia reconstruction using tissue expanders without skin grafts from groin region. Journal of Plastic, Reconstructive \& Aesthetic Surgery.2014; 67:1481-1487 
Fig 1. Extended retro-auricular flap prelaminated with tissue expansion (red- incision; ---separating level and area; blue - the capsule).

(a). the expanded mastoid skin (b). the surrounding scalp was dissected subcutaneously. 


$$
2
$$


Fig 2 Total auricular reconstruction with extended prefabricated retro-auricular flap

1. Design the flap area and incision line. 2. Prepare the extended and prelaminated retro-auricular flap. 3. Insert the cartilage framework into the capsule, and apply negative pressure drainage. 4. Ear lobe translocation. 5. Form cranio-auricular sulcus. 


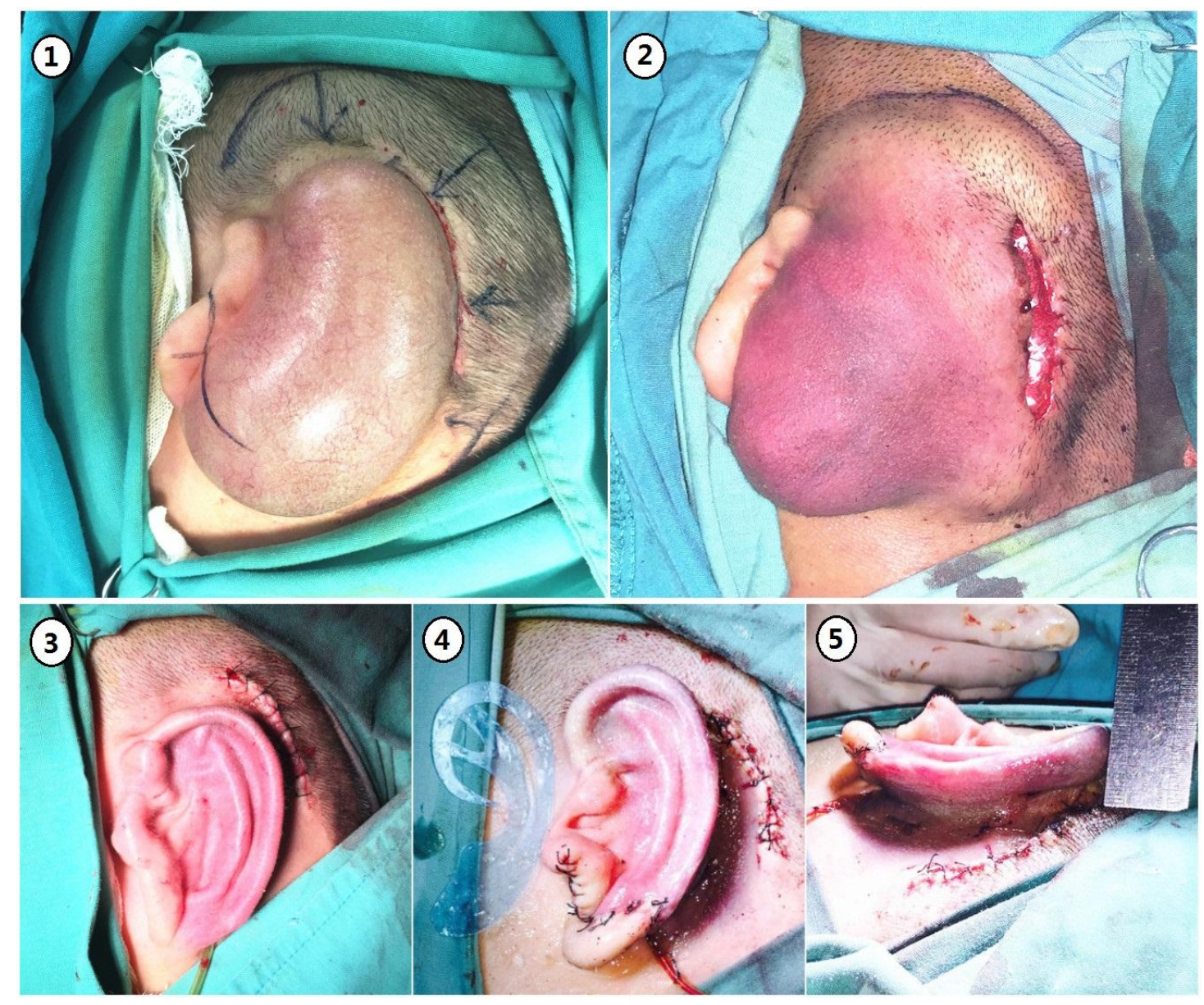


Fig 3. Fabricating the 3-dimensional cartilage framework 


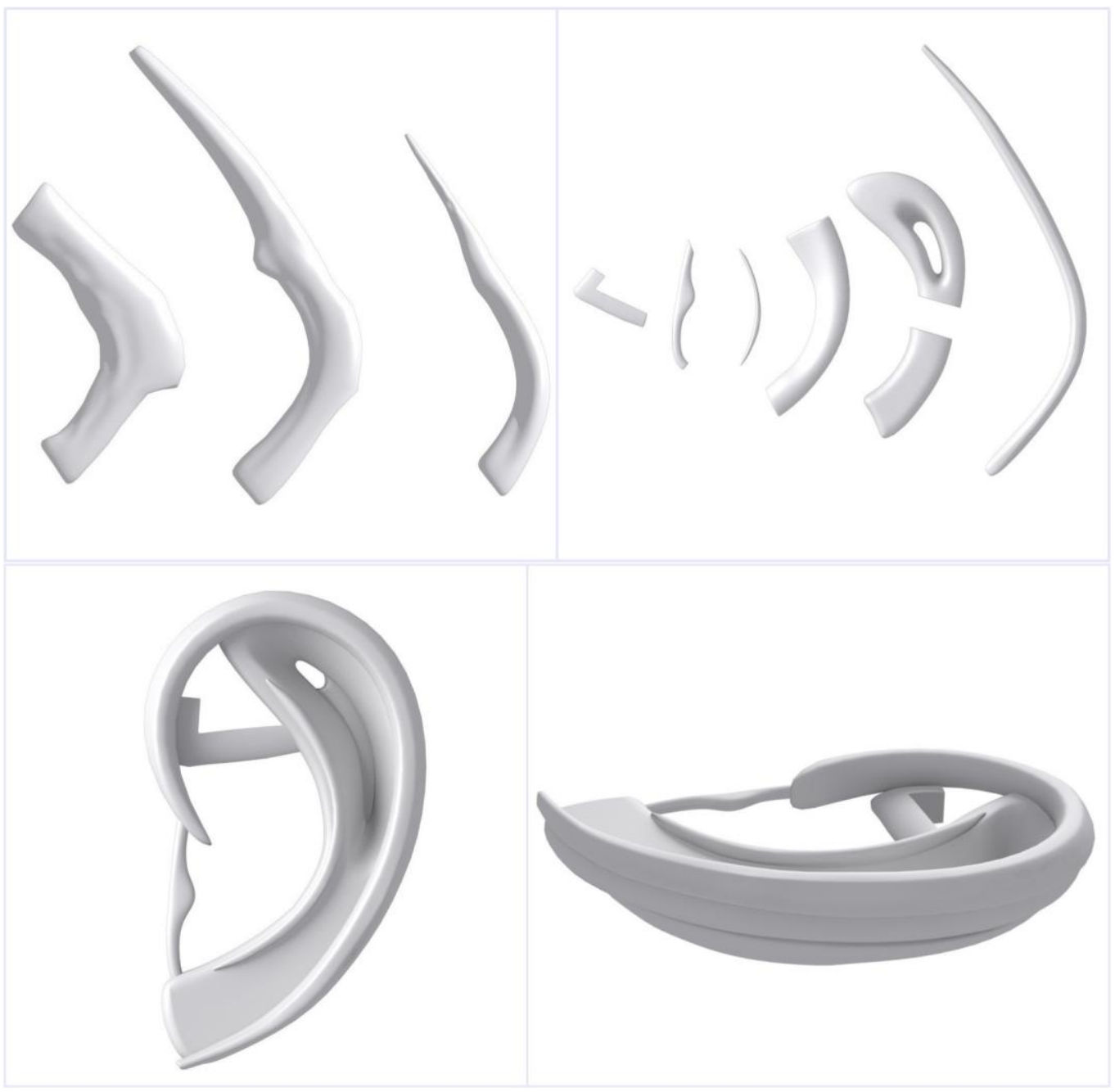


Fig 4. Case 1 A 6-year-old boy presented with microtia (Right, Marx classification IV) 


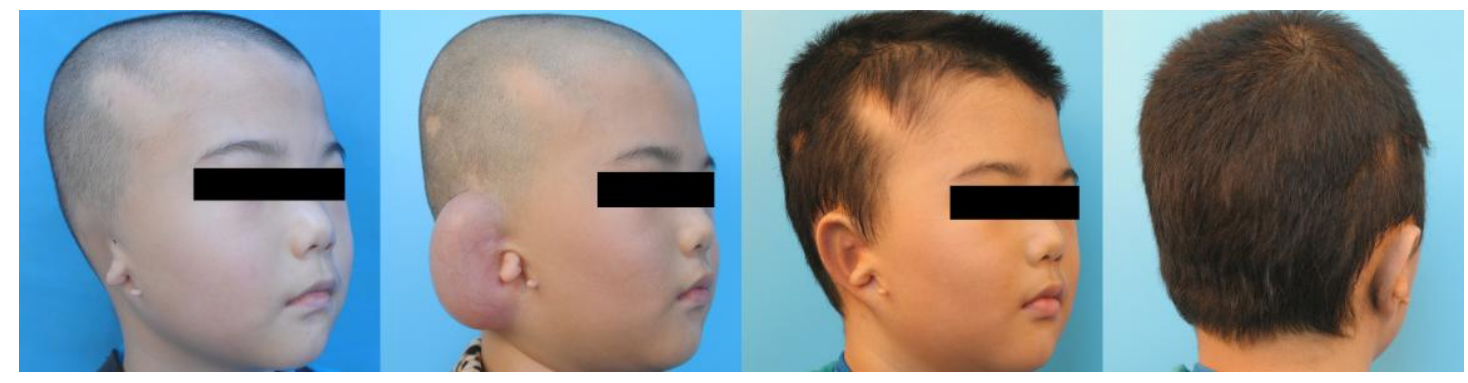


Fig 5. Case 2 A 26 year old woman presented with microtia (Right, Marx classification III) 


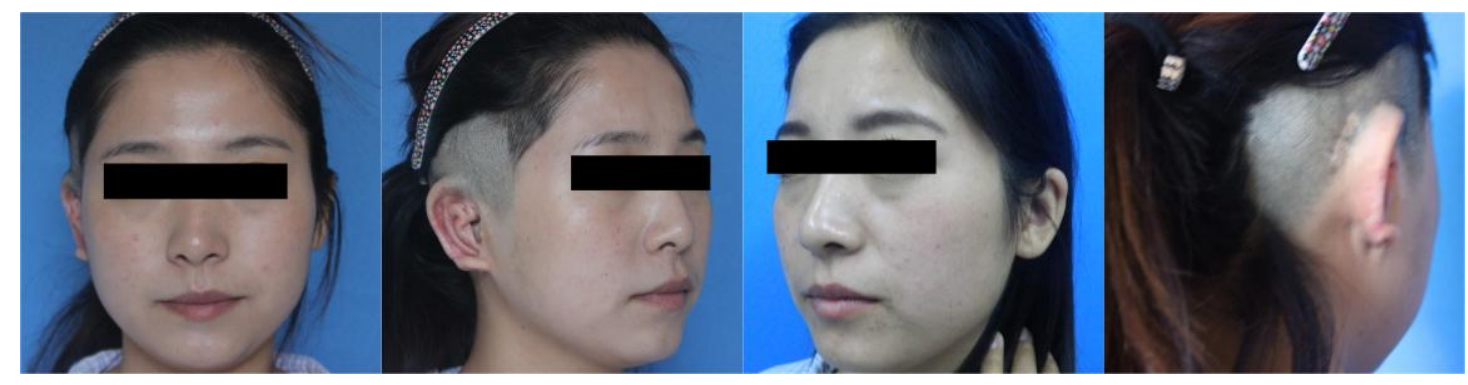


Fig 6. Case 3 A 21 year old woman presented with microtia (Right, Marx classification III) 


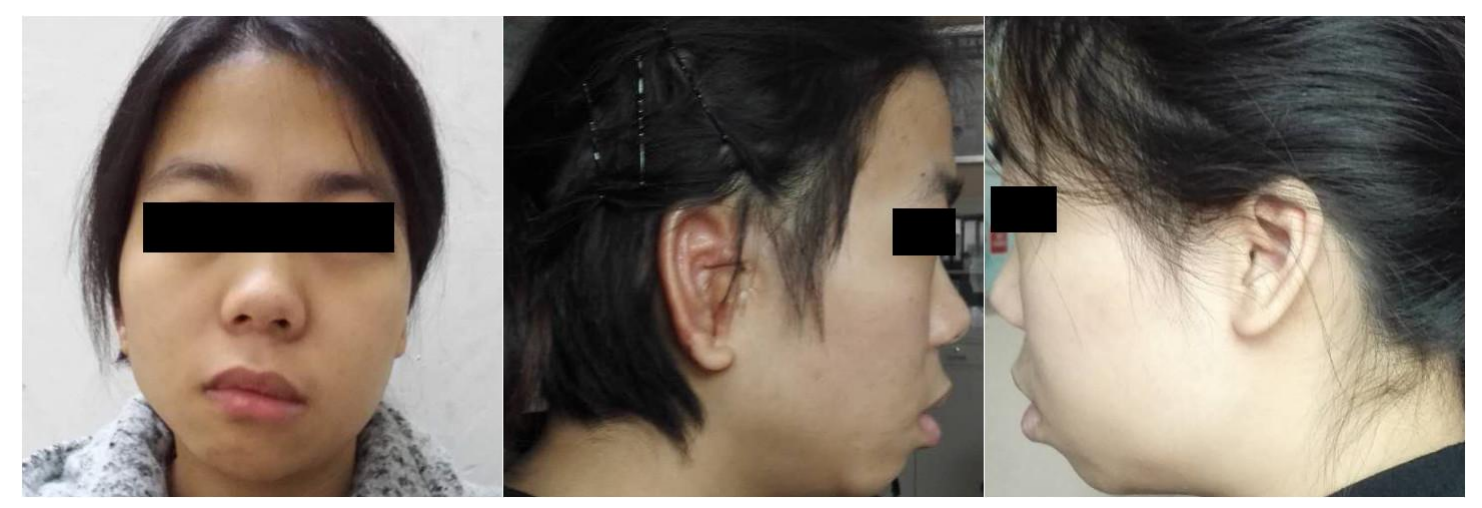


Fig 7. (Left)Skin loss over the left ear helix after bilateral ear reconstruction, repaired with post-auricular fascia, local double pedicle flap and skin grafts. (Right) 3 months after skin loss repairing. 


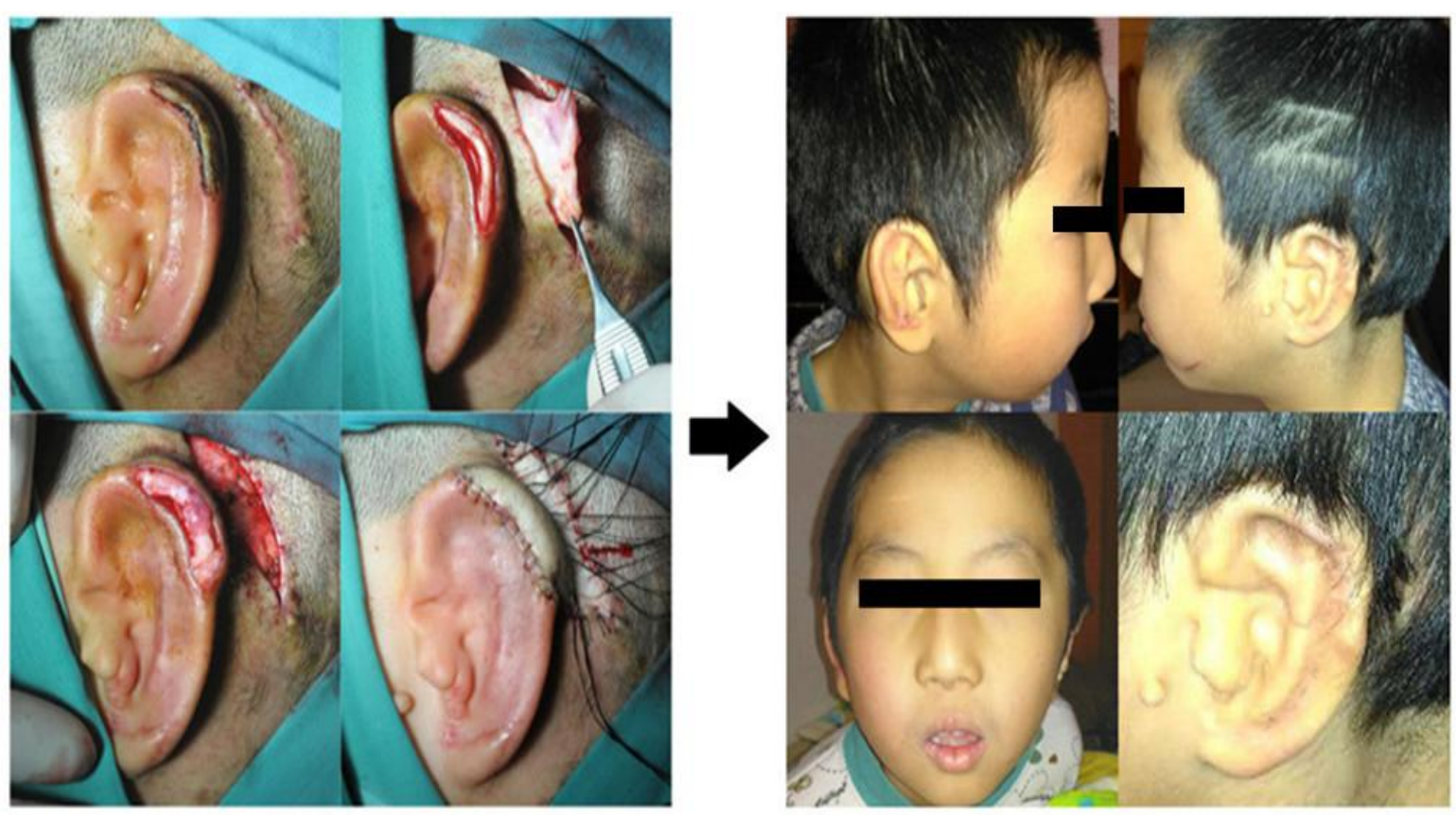


Fig 8. "C" shaped dressing 


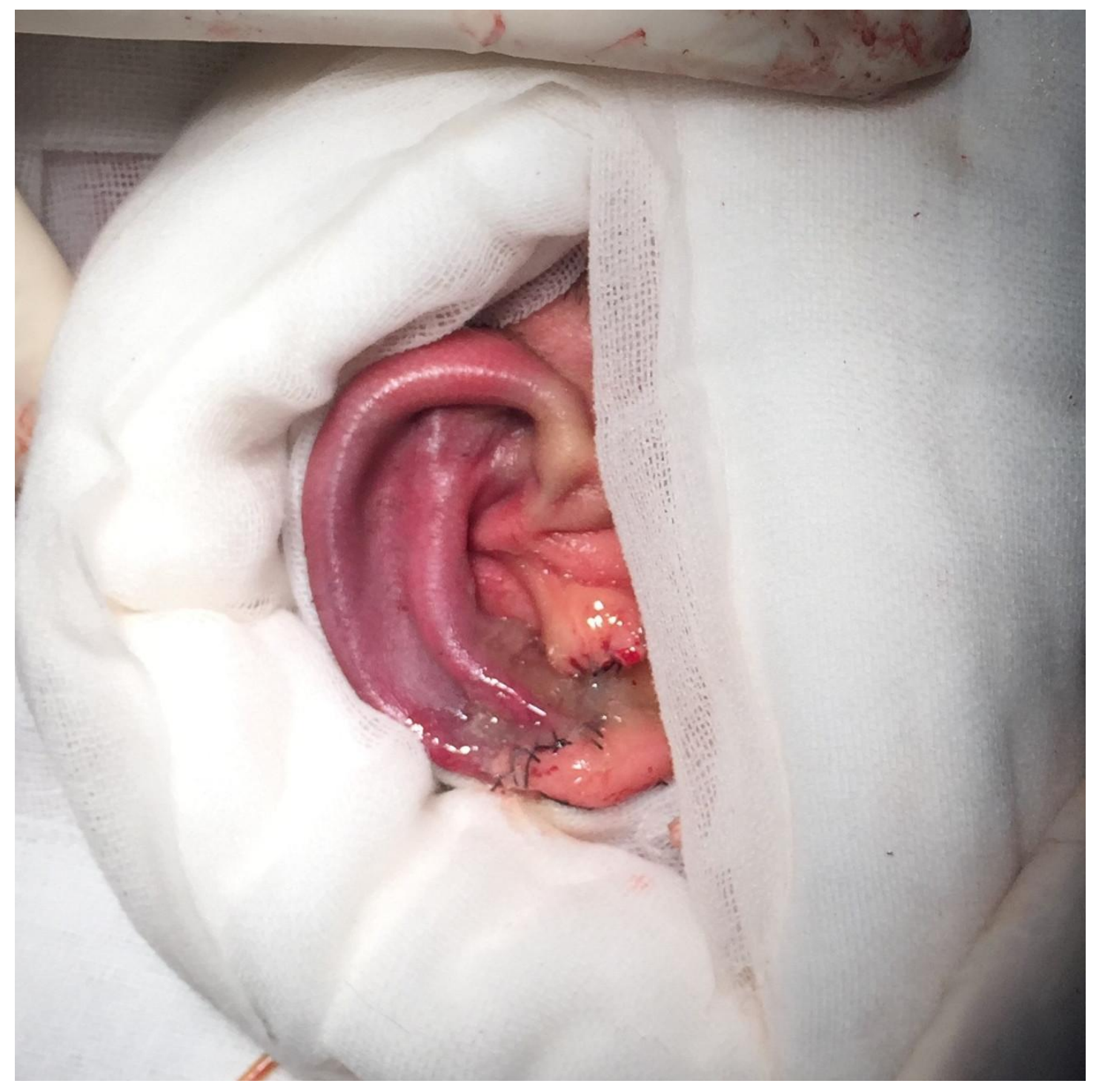


Fig 9. 2 cases of tissue expander exposure treated by debridement and suture during ear construction 

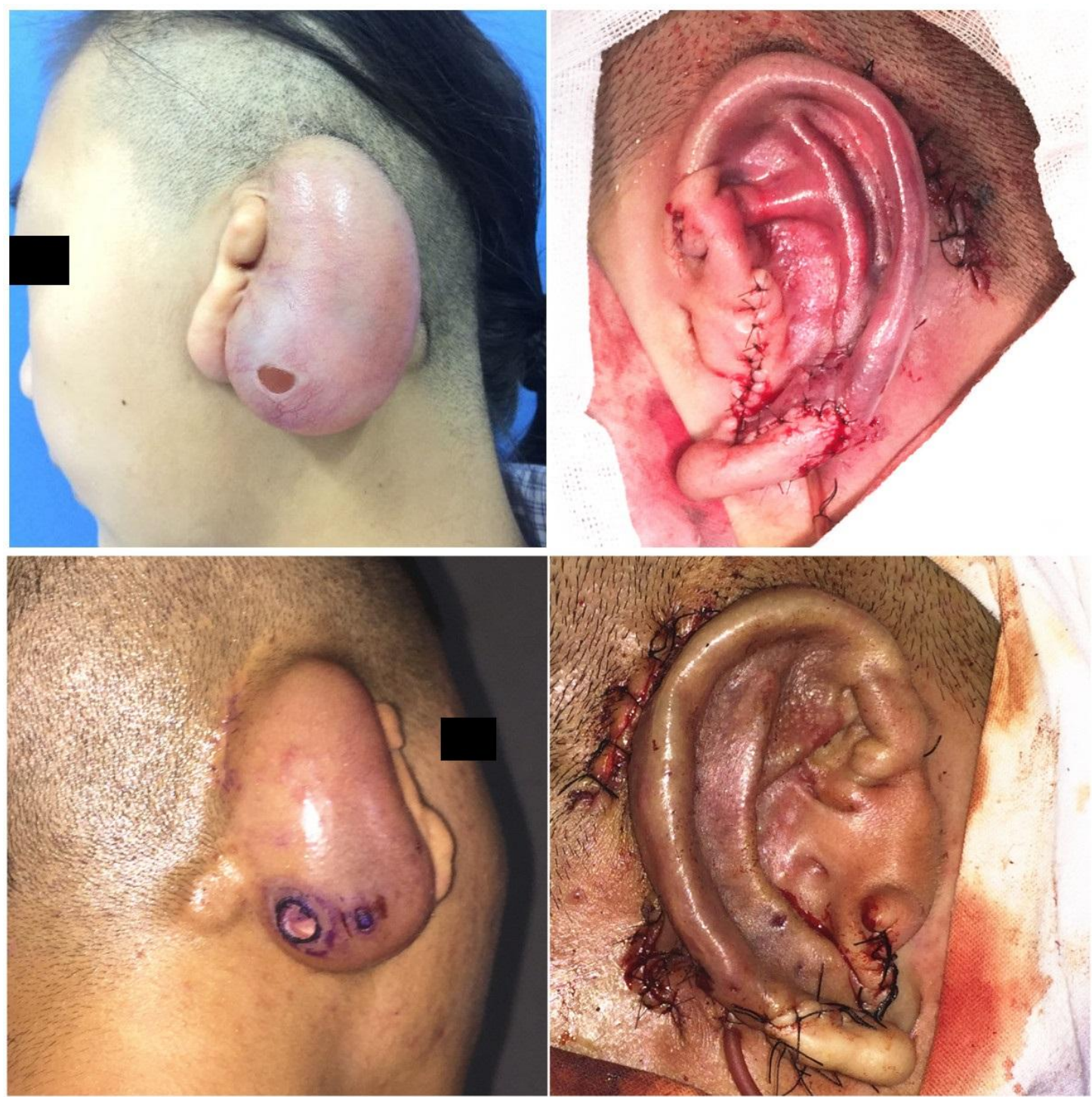
Table 1. Patient data

\begin{tabular}{llllll}
\hline \multicolumn{2}{l}{ Sex of patient(\%) } & \multicolumn{2}{l}{ Type of microtia (\%) } & \multicolumn{2}{l}{ Patient age range(\%) } \\
\hline Male & $23(63.9 \%)$ & Right & $18(50 \%)$ & $5-12$ & $23(63.9 \%)$ \\
Female & $13(36.1 \%)$ & Left & $14(38.9 \%)$ & $13-18$ & $9(25 \%)$ \\
& & Bilateral & $4(11.1 \%)$ & $19-30$ & $4(11.1 \%)$ \\
\hline
\end{tabular}


Table 2. Complications after surgical procedures

\begin{tabular}{llll}
\hline Surgical procedure & Complications & Number of patients & Percentage(\%) \\
\hline Tissue expander insertion & Wound & 3 & $8.3 \%$ \\
Ear reconstruction & Partial loss of skin graft & 2 & $5.6 \%$ \\
\hline
\end{tabular}

